\title{
Pressure-induced energy level crossings and narrowing of photoluminescence linewidth in self-assembled InAIAs/AIGaAs quantum dots
}

\author{
J. Phillips and P. Bhattacharya ${ }^{a)}$ \\ Department of Electrical Engineering and Computer Science, University of Michigan, \\ Ann Arbor, Michigan 48109 \\ U. Venkateswaran \\ Department of Physics, Oakland University, Rochester, Michigan 48309
}

(Received 5 October 1998; accepted for publication 14 January 1999)

We present a study of the hydrostatic-pressure dependence of the photoluminescence from $\mathrm{In}_{0.5} \mathrm{Al}_{0.5} \mathrm{As} / \mathrm{Al}_{0.25} \mathrm{Ga}_{0.75} \mathrm{As}$ self-assembled quantum dots. Three distinct regions of quantum-dot peak-energy shift with pressure are observed and are attributed to energy level crossings and band mixing effects. In addition, a large reduction in photoluminescence linewidth with applied pressure is noted. (C) 1999 American Institute of Physics. [S0003-6951(99)01211-5]

Self-assembled quantum dots have proven to be an excellent method in realizing quasizero-dimensional structures through nearly conventional epitaxial growth techniques. ${ }^{1-3}$ The quantum-dot formation is dependent on both epitaxial growth kinetics and the strain in the system. ${ }^{4}$ The band structure of these quantum dots needs to be better understood to properly design quantum-dot devices. One technique used to obtain deformation potentials in strained systems and information on band structure is measurement under applied pressure. Itskevich et al. ${ }^{5}$ have studied self-assembled InAs/ GaAs quantum dots under applied pressure and have found evidence of a $\Gamma-X$ crossover and type-II band alignments. Here we report a study of the photoluminescence of $\mathrm{In}_{0.5} \mathrm{Al}_{0.5} \mathrm{As}_{\mathrm{Al}} / \mathrm{Al}_{0.25} \mathrm{Ga}_{0.75} \mathrm{As}$ self-assembled quantum dots under hydrostatic pressure in the effort to further understand the band structure and strained nature of the quantum dots.

Heterostructures for photoluminescence (PL) and atomic force microscopy (AFM) measurements were grown by molecular beam epitaxy. The grown structure consists of a GaAs buffer layer followed by a $200 \mathrm{~nm} \mathrm{Al}_{0.25} \mathrm{Ga}_{0.75} \mathrm{As}$ barrier, both grown at $630^{\circ} \mathrm{C}$. The $\mathrm{In}_{0.5} \mathrm{Al}_{0.5} \mathrm{As}$ quantum dots were then grown at $540^{\circ} \mathrm{C}$ with a nominal thickness of $10 \mathrm{ML}$. The quantum-dot growth was monitored by reflection high energy electron diffraction and three-dimensional island growth was found to commence after $\sim 8 \mathrm{ML}$, which is in agreement with other reports. ${ }^{6}$ A 15 s growth interruption at this point allowed further dot formation and was followed by the growth of $200 \mathrm{~nm}$ of $\mathrm{Al}_{0.25} \mathrm{Ga}_{0.75} \mathrm{As}$ at $630^{\circ} \mathrm{C}$. A layer of $\operatorname{In}_{0.5} \mathrm{Al}_{0.5}$ As quantum dots was then grown on the surface under conditions similar to the buried dots for AFM measurements, which reveal a density of $\sim 1 \times 10^{11}$ dots $/ \mathrm{cm}^{2}$. In addition, a reference $\mathrm{Al}_{0.25} \mathrm{Ga}_{0.75} \mathrm{As}$ sample was grown under similar conditions, but without the quantum-dot layers.

Photoluminescence measurements were performed using an $\operatorname{Ar}^{+}(\lambda=488 \mathrm{~nm})$ laser, a $1 \mathrm{~m}$ monochromator, and a photomultiplier with photon counting. Excitation was less

${ }^{a)}$ Electronic mail: pkb@eecs.umich.edu than $30 \mathrm{~mW}$ for all measurements with a diameter of $\sim 50 \mu \mathrm{m}$, corresponding to a region containing an estimated $2 \times 10^{6}$ dots. A Merrill-Bassett-type diamond anvil cell and a closed cycle helium cryostat were used for the high pressure measurements at low temperature. A 4:1 methanolethanol solution served as the pressure transmitting medium and the pressure calibration was done using fluorescence from a small piece of ruby mounted with the sample. Pressure was adjusted at room temperature for all measurements.

The low temperature PL spectra of the quantum-dot sample for varying pressures are shown in Fig. 1. At 1 bar, three peaks at $E=1.5,1.705$, and $1.845 \mathrm{eV}$ are observed corresponding to luminescence from the GaAs buffer, InAlAs quantum dots, and AlGaAs bulk, respectively. The PL peak energy shifts with pressure are shown in Fig. 2. The underlying GaAs buffer peak was observed for all pressure

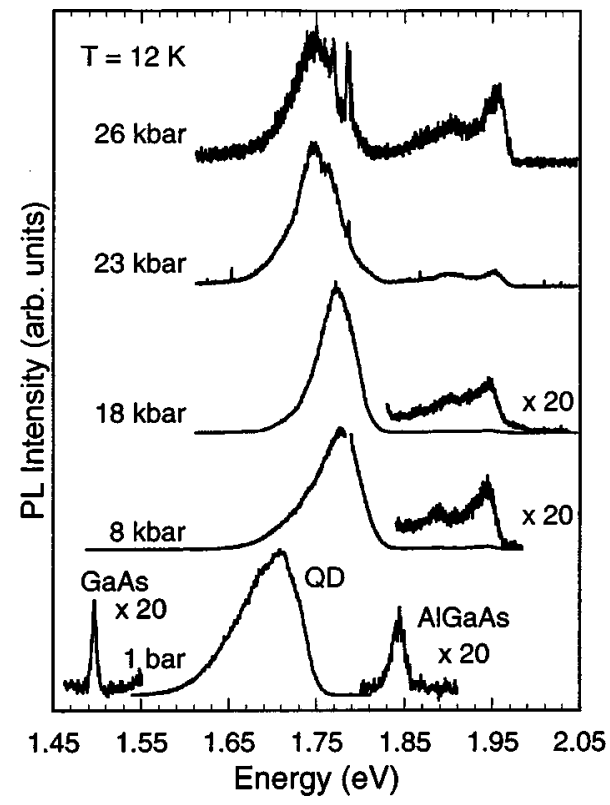

FIG. 1. Photoluminescence from the quantum-dot sample for various pressures showing luminescence peaks for the InAlAs quantum dots, AlGaAs barrier, and $\mathrm{GaAs}$ buffer. 


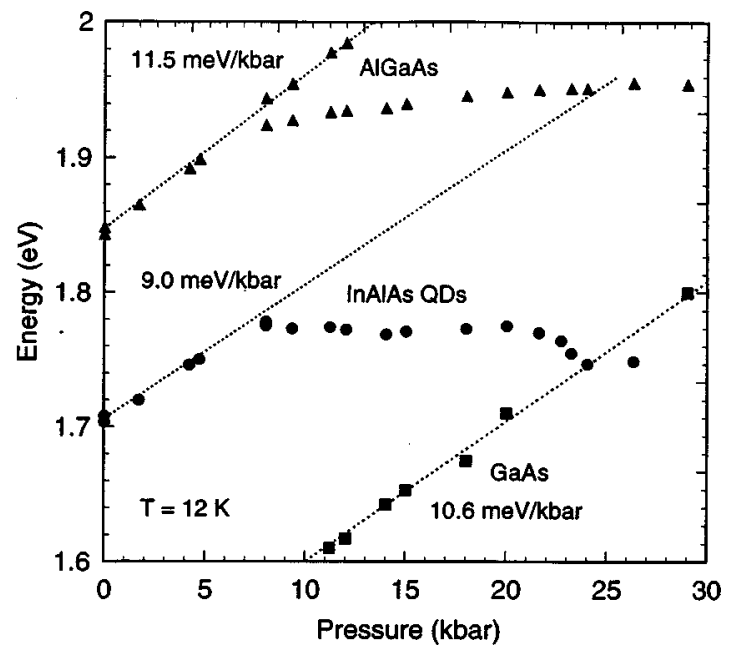

FIG. 2. Photoluminescence peak intensity shift with pressure for the quantum-dot sample. GaAs data points are not shown between 22 and 28 kbar to avoid confusion with quantum-dot data.

measurements, with a linear pressure coefficient of 10.6 $\mathrm{meV} / \mathrm{kbar}$, serving as a good reference for verifying pressure calibration. Other PL peaks shift linearly with applied pressure up to $8 \mathrm{kbar}$ with pressure coefficients for AlGaAs and InAlAs quantum dots of 11.5 and $9.0 \mathrm{meV} / \mathrm{kbar}$, respectively. The pressure coefficient of InAlAs quantum dots is smaller than that expected for InAlAs bulk, but is consistent with that reported for self-assembled InAs/GaAs quantum dots. ${ }^{5}$ We believe that the reduced pressure shift is due to the highly confined energy levels in the dots, similar to the reduced pressure coefficients reported for increasing confinement in InGaAs/AlGaAs quantum wells. ${ }^{7}$ Between 8 and 20 kbar, there is no significant energy shift (or a small redshift of $\sim 1 \mathrm{meV} / \mathrm{kbar}$ ) in the quantum-dot peak with pressure. Above $20 \mathrm{kbar}$, there is a gradual redshift in peak energy with pressure, and finally the quantum dot luminescence is quenched above $26 \mathrm{kbar}$. The true cause of the photoluminescence peak shift with pressure cannot be deduced from these measurements alone; however, a possible explanation is given below and is schematically shown in Fig. 3 for the three distinct pressure regions.

The energy states in self-assembled quantum dots are quite different from those in the corresponding bulk material because of quantum confinement and the built-in strain between the quantum dot and barrier material. Application of external pressure modifies the strain field in and around the quantum dots due to the differences in the bulk moduli of the two materials. In addition, pressure-induced energy level crossings also influence the pressure coefficient of the quantum-dot PL peak. Due to the much larger hole effective mass, the valence band states are not expected to shift significantly with pressure as compared to conduction band states. Therefore, only conduction band states are considered in this discussion. From the known composition dependence of the $\Gamma$ and $X$ conduction band energies in ternary alloys, it is seen that the $\Gamma$ and $X$ conduction bands in InAlAs/AlGaAs have type-I and type-II band alignments, respectively, for all values of the valence band offset. Theoretical calculations of the strain tensor in these quantum $\operatorname{dots}^{8}$ predict a large degree of built-in biaxial strain which may lift the degeneracy of the $X_{z}$ and $X_{x y}$ bands, with the $X_{x y}$ ground state lower in

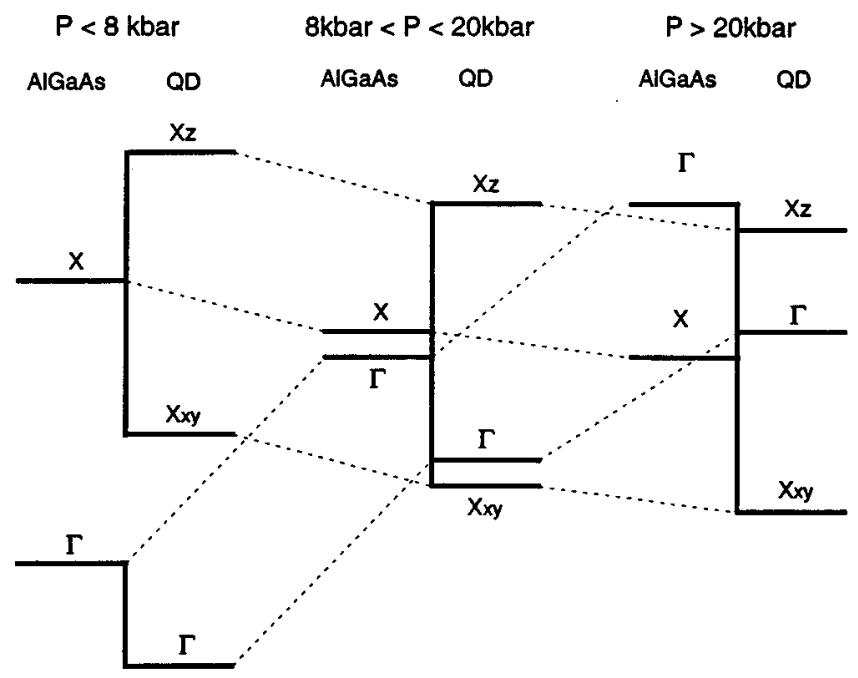

FIG. 3. Schematic of proposed band alignment for quantum dots and AlGaAs barriers for the three pressure regions observed. The levels denote the AlGaAs band edge or quantum confined ground state in the quantum dots.

energy than that of $X_{z}$. There is also a strong built-in hydrostatic pressure component. It is therefore possible to have a type-I alignment for $X_{x y}$ and type-II for $X_{z}$ energy levels. The pressure coefficient of $9.0 \mathrm{meV} / \mathrm{kbar}$ indicates that the deformation potential of the quantum dot is not very different from bulk material. This result will be analyzed in detail. We believe that the change in the pressure coefficient at 8 kbar results for the crossover between the quantized levels in the $\Gamma$ and $X_{x y}$ bands in the quantum dots, as shown in Fig. 3. After this crossover, we believe that the observed PL originates from the $X_{x y}$ levels which are the low energy states for $P>8$ kbar. The significantly reduced pressure shift of the PL supports that the PL is from $X$ conduction band transitions. This explanation suggests that there exists an efficient scattering mechanism that allows a fast relaxation from $\Gamma$ to $X_{x y}$ and an efficient radiative recombination between $X_{x y}$ and hole states in the quantum dots. The recombination can be defect assisted, impurity assisted, or phonon mediated. It should be noted that the peak intensity of the quantum-dot luminescence remains fairly constant up to $\sim 18 \mathrm{kbar}$ and decreases significantly thereafter, as shown in Fig. 4. Additionally, above $18 \mathrm{kbar}$, we observe a redshift in peak energy position with pressure which we believe is due to the onset of a crossover between $\Gamma$ states in the quantum dots and $X$ states in the AlGaAs barrier. Extrapolation of the initial 9 $\mathrm{meV} / \mathrm{kbar}$ shift of the quantum-dot peak intersects the observed $X$ state for the AlGaAs near $23 \mathrm{kbar}$, supporting this explanation. The mixing of the $\Gamma$ and $X$ bands causes an anticrossing behavior, resulting in a redshift similar to that observed for InAs/GaAs quantum dots grown on misoriented substrates. ${ }^{9}$ The PL peak intensity additionally becomes weak after this crossover $(>26 \mathrm{kbar})$ since the transition becomes indirect in both real and $k$ space. In addition, attention should be given to the fact that radiative transitions between $X$ states and hole states should be dominated by phonon replicas. The quantum-dot PL in these measurements is particularly broad and therefore does not clearly show phonon replicas in regions where we suggest $X$ state transitions. For the 


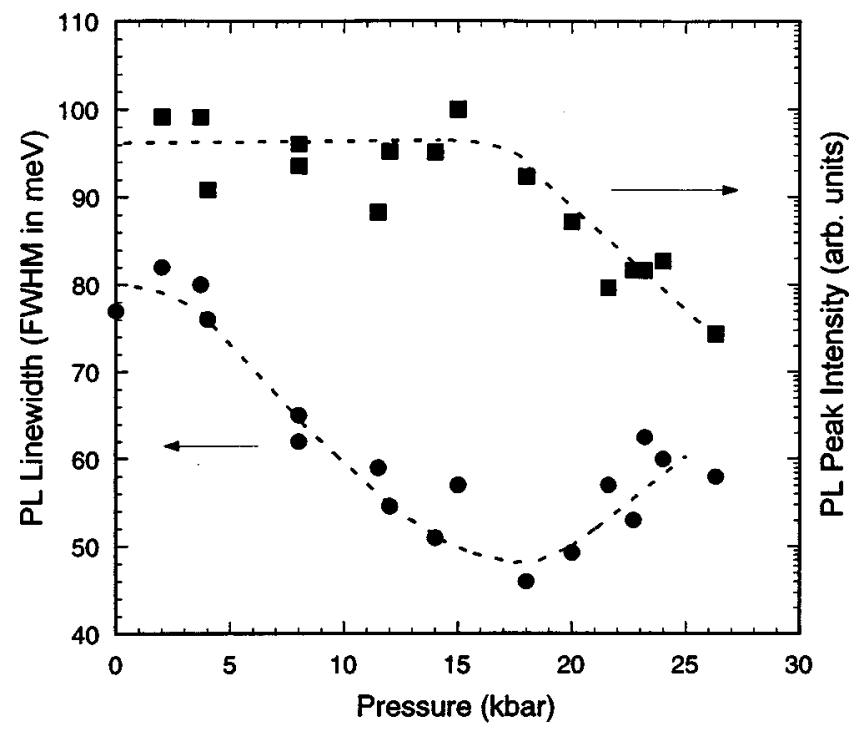

FIG. 4. Photoluminescence linewidth (full width at half maximum) and peak intensity as a function of applied pressure.

AlGaAs barrier material, we begin to see features from phonon replicas above $8 \mathrm{kbar}$, as shown in Fig. 1. These features are not particularly clear due to the weak luminescence from the barrier material. However, these features are observed very clearly in the reference AlGaAs sample.

In addition to the PL peak energy shift with pressure, we observe unique features in the PL linewidth with applied pressure, as shown in Fig. 4. The PL linewidth remains fairly constant up to a few kilobars and then decreases significantly ( $\sim 45 \%)$ between 5 and 18 kbar. We attribute the large reduction in PL linewidth to varying pressure coefficients for dots of different size and hence different energy levels. At the lowest applied pressures, asymmetric quantum-dot PL spectra are observed with a significant low energy tail corresponding to dots of larger size. For increasing applied pressure, the spectra become more symmetric. We believe that the dots contributing to the lower energy luminescence, whose energy levels are less confined, have a larger pressure coefficient, similar to the case for quantum wells. ${ }^{7}$ As a result, this causes the ground state energies of the quantum dots to merge, resulting in a reduced PL linewidth. A pressure-induced reduction in PL linewidth observed earlier in quantum wells has been attributed to a pressure-induced decrease in carrier density. ${ }^{10}$ Carrier density decrease may contribute to the observed quantum-dot PL linewidth decrease. However, a carrier density decrease does not explain the disappearance of the low energy tail with increasing pressure, but instead will be expected to cause a reduction in luminescence on the high energy side. An increase in the PL linewidth observed above $18 \mathrm{kbar}$ can be attributed to the $\Gamma-X$ energy band crossover.

In conclusion, we have shown that self-assembled $\mathrm{In}_{0.5} \mathrm{Al}_{0.5} \mathrm{As} / \mathrm{Al}_{0.25} \mathrm{Ga}_{0.75} \mathrm{As}$ quantum dots exhibit unique changes in PL spectra under applied pressure. The PL peak energy shows three distinct regions with different pressure coefficients which are attributed to energy level crossovers. Further experiments involving theoretical calculation and temperature-dependent measurements should more clearly reveal the transitions involved at varying applied pressure. In addition, the photoluminescence linewidth shows a significant decrease with applied pressure which we believe is due to varying pressure coefficients for dots of different size.

This work was supported by the National Science Foundation under Grant No. ECS-9628973 and the Army Research Office under Grant No. DAAG 55-97-1-0156. One of the authors (U.V.) acknowledges partial support from the Donors of the Petroleum Research Fund, administered by the American Chemical Society.

${ }^{1}$ L. Goldstein, F. Glas, J. Y. Marzin, M. N. Charasse, and G. LeRoux, Appl. Phys. Lett. 47, 1099 (1984).

${ }^{2}$ P. Berger, K. Chang, P. Bhattacharya, J. Singh, and K. Bajaj, Appl. Phys. Lett. 53, 684 (1988).

${ }^{3}$ See the following and references therein, D. Bimberg, M. Grundmann, and N. N. Ledentsov, Mater. Res. Bull. 23, 31 (1998).

${ }^{4}$ N. P. Kobayashi, T. R. Ramachandran, P. Chen, and A. Madhukar, Appl. Phys. Lett. 68, 3299 (1996).

${ }^{5}$ I. E. Itskevich, S. G. Lyapin, I. A. Troyan, P. C. Klipstein, L. Eaves, P. C. Main, and M. Henini, Phys. Rev. B 58, R4250 (1998).

${ }^{6}$ R. Leon, S. Fafard, D. Leonard, J. L. Merz, and P. M. Petroff, Appl. Phys. Lett. 67, 521 (1995).

${ }^{7}$ V. A. Wilkinson, A. D. Prins, D. J. Dunstan, L. K. Howard, and M. T. Emeny, J. Electron. Mater. 20, 509 (1991).

${ }^{8}$ H. Jiang and J. Singh, Phys. Rev. B 56, 4696 (1997).

${ }^{9}$ G. H. Li, A. R. Goni, K. Syassen, O. Brandt, and K. Ploog, Phys. Rev. B 50, 18420 (1994).

${ }^{10}$ S. Ernst, A. R. Goni, K. Syassen, and K. Eberl, Phys. Rev. Lett. 72, 4029 (1994). 